\title{
HARMONIC DECOMPOSITION OF ORBIT DATA FOR MULTIPOLE ANALYSIS ${ }^{\dagger}$
}

\author{
M.J. Yang ${ }^{\ddagger}$, Fermilab, Batavia, IL 60510, USA
}

\begin{abstract}
This paper describes a simple analysis procedure that transforms a set of beamline orbit data into a set of harmonic orbits of first, second, and third order or higher. Each harmonic orbit can be studied individually to identify errors of the specific order with minimum interference from other orders. Effectively these are orbits caused by kicks, due to harmonic errors, propagated through linear lattice. Examples from accelerator study will be presented. The application and inherent limitations of this analysis procedure are discussed.
\end{abstract}

\section{INTRODUCTION}

Orbit excitation is typically the means with which linear beamline optics is studied, be it lattice function measurement or transverse coupling. The existences of higher order elements often make the analysis more complicated as they either cause higher order coupling effects or change focusing property. Unlike transfer lines, where usage of non-linear elements is limited, circular machines require the use of sextupoles extensively to control the chromaticity. In Figure 1 are examples of horizontal plane orbit data as a function of voltage on the Booster extraction kicker. With kicker being in the vertical plane any horizontal position variation is an indication of skew coupling error and, in this example, it is clearly not linear.
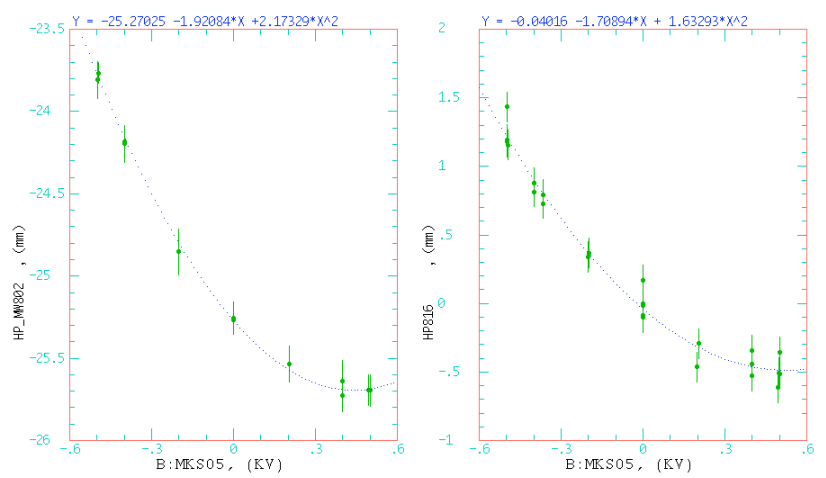

Figure 1. Horizontal beam position data versus Booster vertical extraction kicker voltage. Plotted here are position data from profile monitor MW802 and BPM HP816 in the MI8 line.

Other sources of first order errors, such as rolled corrector dipoles or rolled Beam Position Monitors (BPMs), can interfere with coupling study. Improperly scaled corrector currents may be confused for focusing errors. With harmonic decomposition these types of errors are left with first order orbits and not with higher order orbits.

This procedure has been applied in the optics study of the Fermilab Booster to Main Injector 8-GeV (MI8) trans-

\footnotetext{
$\dagger$ Work supported by the US Department of Energy under contract DE-AC02-76CH00300.

† E-mail: YANG@FNAL.GOV
}

fer line and in the coupling study of Fermilab Recycler Ring. Examples of study results will also be presented.

\section{PRINCIPLE}

The idea of harmonic decomposition hinges on the assumption that orbit perturbation due to harmonic errors is small and its cascading effect can be ignored, i.e. kick error is propagated on a linear lattice without incurring further effect from harmonic errors. A good assumption when errors are small and infrequent.

With linear lattice the position response at $x_{i}$, the $i$-th location with multipole error, can be given by

$$
x_{i}=\sum_{k} f(i, k) \cdot \theta_{k},
$$

where $\theta_{k}$ is kick angle at the $k$-th corrector dipole location and the summation includes all corrector dipoles used in the study. The response function $f(i, k)$ is defined as

$$
f(i, k)=\frac{\sqrt{\beta_{i} \beta_{k}} \cos \left(\phi_{i}-\phi_{k}\right)}{2 \sin (\pi \cdot Q)}
$$

for circulating beam closed orbit and is defined as

$$
f(i, k)=\left\{\begin{array}{cc}
\sqrt{\beta_{i} \beta_{k}} \sin \left(\phi_{i}-\phi_{k}\right) & \langle i \text { downstream of } k\rangle \\
0 & \text { otherwise }
\end{array}\right.
$$

for transfer line or first turn orbit. The symbol $Q$ in (1a) denotes tune of a circular machine. Both $i$ and $k$ are indices to locations in the beamline and $f(i, k)$ is zero if they point to locations not of the same plane.

In actual beam study kicks applied at multiple locations are most often correlated. An angle $\theta$, that is representative of the magnitude of orbit displacement, can be chosen such that $\theta_{k}=\alpha_{k} \cdot \theta$. The kick-plane response can then be expressed as $x_{i}=g_{i} \cdot \theta$, with

$$
g_{i}=\sum_{k} f(i, k) \cdot \alpha_{k}
$$

In the case of closed 3-bump study $\theta$ would be the kick from the first corrector dipole and $g_{i}$ would be zero every where except within the bump.

The total kick error $\vartheta_{i}$ due to harmonic errors at the $i$-th location is:

$$
\vartheta_{i}=\sum_{n} I_{i, n} \cdot x_{i}^{n}=\sum_{n} I_{i, n} \cdot\left(g_{i} \cdot \theta\right)^{n}=\sum_{n} I_{i, n} \cdot g_{i}^{n} \cdot \theta^{n}
$$

where $I_{i, n}=\frac{B_{i, n} \cdot L_{n}}{n !}$ is the integrated strength of $n$-th order multipole. For each order of $n$ the choice of $B_{i, n}$ would be either the normal or the skew component, depending on the kicked plane and the plane $\vartheta_{i}$ is in. The geometrical factor dictates the plane a given multipole can contribute, while its orthogonal counterpart contributes to the other. 
This fact is utilized in deriving equation (3) which shows only components with non-zero geometric factor $g_{i}{ }^{n} \cdot \theta^{n}$. The effects of multipoles are summarized in Table I.

The position change at the $j$-th observable location due to harmonic errors at $i$-th location is:

$$
\Delta u_{j, i}=\vartheta_{i} \cdot f(j, i)=f(j, i) \cdot \sum_{n} I_{i, n} \cdot g_{i}^{n} \cdot \theta^{n}
$$

with $u$ being either horizontal or vertical position.

The position at the $j$-th location is the linear response to all $\theta_{k}$ kicks from corrector dipoles used and to kicks from harmonic errors elsewhere:

$$
\begin{aligned}
u_{j} & =h_{j} \cdot \theta+\sum_{i} \Delta u_{j, i} \\
& =h_{j} \cdot \theta+\sum_{i} f(j, i) \cdot \sum_{n} I_{i, n} \cdot g_{i}^{n} \cdot \theta^{n} \\
& =h_{j} \cdot \theta+\sum_{n} \theta^{n} \cdot \sum_{i} f(j, i) \cdot I_{i, n} \cdot g_{i}^{n} \\
& =h_{j} \cdot \theta+\sum_{n} \theta^{n} \cdot c_{j, n}
\end{aligned}
$$

where

$$
c_{j, n}=\sum_{i} f(j, i) \cdot k_{i, n} \cdot g_{i}{ }^{n}
$$

The definition of $h_{j}$ is similar to that of $g_{i}$ except that the observation location $j$ can be in either plane. When $u_{j}$ is in the other plane $h_{j}$ becomes zero and equation (5) is further simplified to:

$$
u_{j}=\sum_{n} \theta^{n} \cdot c_{j, n}
$$

In a 3-bump study both $h_{j}$ and $g_{i}$ are zero every where except within the bump and the summation in equation (5a) is reduced to only a few locations.

Equation (5) gives the basics of harmonic decomposition. It says that the position response at $j$-th location is a polynomial function of $\theta$. Each coefficient $c_{j, n}$ is the sum of effects from kicks, due to $n$-th order error everywhere, propagated to $j$-th location on a linear lattice.

During machine studies data is usually taken with respect to the nominal orbit, not to the center of multipole fields. The strength $I_{i, n}$ in Equation (5), therefore, should include feed-down effects from all higher orders.

\section{APPLICATIONS}

\section{Extracting harmonic orbit}

The result of second order polynomial fit to MI8 line data is shown in Figure 1 as dotted blue line and as text at the top. Similar data from the Fermilab Recycler Ring study is shown in Figure 2, with third order polynomial fit and with vertical scale in $10 \mathrm{~s}$ of microns, instead of $\mathrm{mm}$. While the MI8 line data in Figure 1 does not appear to have third order content, the data in Figure 2 can not be described with a second order polynomial.

When using polynomial coefficients of a specific order as positions in the harmonic orbit it is important that proper unit is assigned. In Figure 2 the polynomial fit is with respect to current reading of corrector dipole H400 and the units, therefore, are $\mathrm{mm} / \mathrm{amp}$ for first order, $m m / a m p^{\wedge} 2$ for second order, and so on.
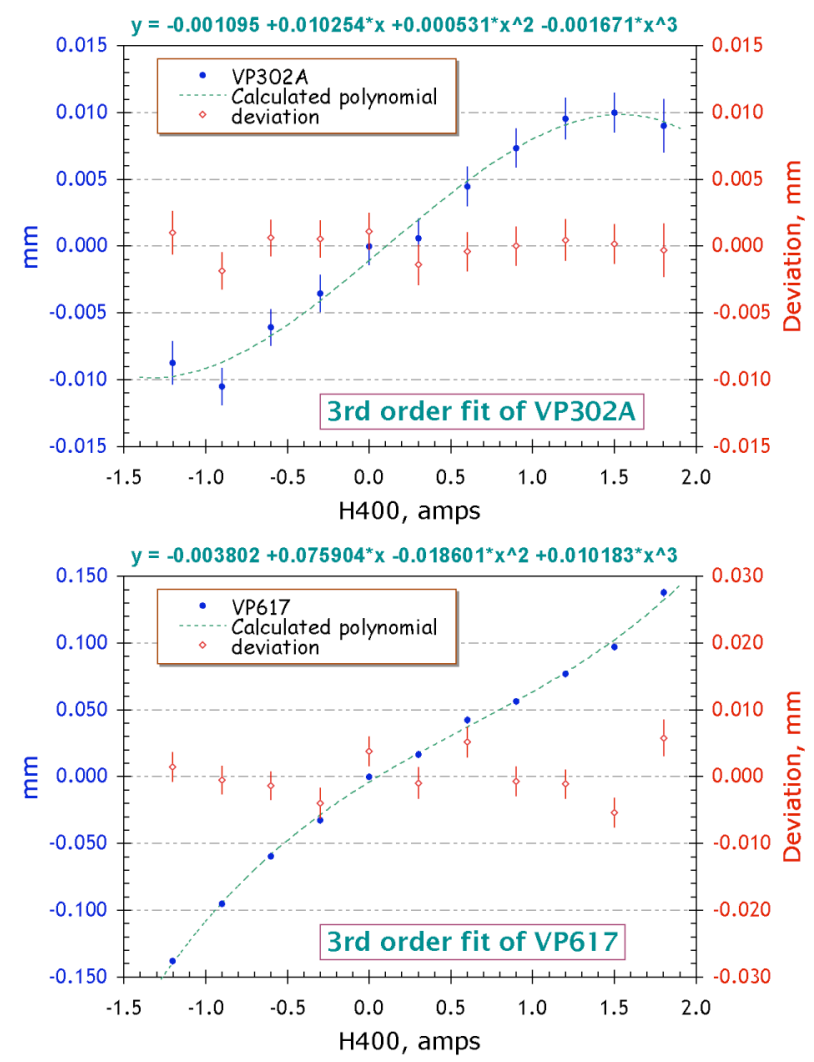

Figure 2. Vertical plane position as a function of horizontal 3-bump H402, at VP302A and VP617 locations. H400 current reading is shown as horizontal axis.

Table I Kick errors due to magnetic multipoles.

\begin{tabular}{|l|l|l|}
\hline $\begin{array}{l}\text { Harmonic } \\
\text { components }\end{array}$ & $\begin{array}{l}\text { Orbit } \\
\text { Horizontal }\end{array}$ & $\begin{array}{l}\text { Orbit } \\
\text { Vertical }\end{array}$ \\
\hline quadrupole & In-plane & In-plane \\
\hline sextupole & In-plane & Coupling \\
\hline Octupole & In-plane & In-plane \\
\hline Decapole & In-plane & Coupling \\
\hline skew quadrupole & coupling & Coupling \\
\hline skew sextupole & coupling & In-plane \\
\hline skew octupole & coupling & Coupling \\
\hline skew decapole & coupling & In-plane \\
\hline
\end{tabular}

Applying harmonic orbit for analysis

A quick reference to the effects of multipole fields is shown in Table I. Depending on the displacement plane and the order of multipole the effect can alternate between kick errors in the same plane and cross-plane coupling. If the purpose is to study the normal sextupole or decapole component vertical plane orbit displacement is desirable. On the other hand, horizontal displacement is the choice for studying skew multipoles. The effect of normal octupole error is always in the plane of primary displacement.

\section{DATA EXAMPLES}

\section{MI8 transfer line}

Figure 3 shows both horizontal and vertical second order 
harmonic orbits from a recent MI8 transfer line study [1]. The purpose was to find evidences of higher order field errors within the extraction septum magnet MP02, of the Fermilab Booster Ring. Using vertical extraction kickers to change beam position through the septum magnet the downstream beamline orbit data was taken and analyzed. A vertical displacement of $5.6 \mathrm{~mm}$ at MP02 is expected for $1 \mathrm{KV}$ change in the extraction kicker setting. In either plane, matching the observed second order harmonic orbit requires placing a single kick at the extraction septum. The calculation, shown in magenta trace, describes a free oscillation downstream of MP02. This is clearly a signature of second order error.

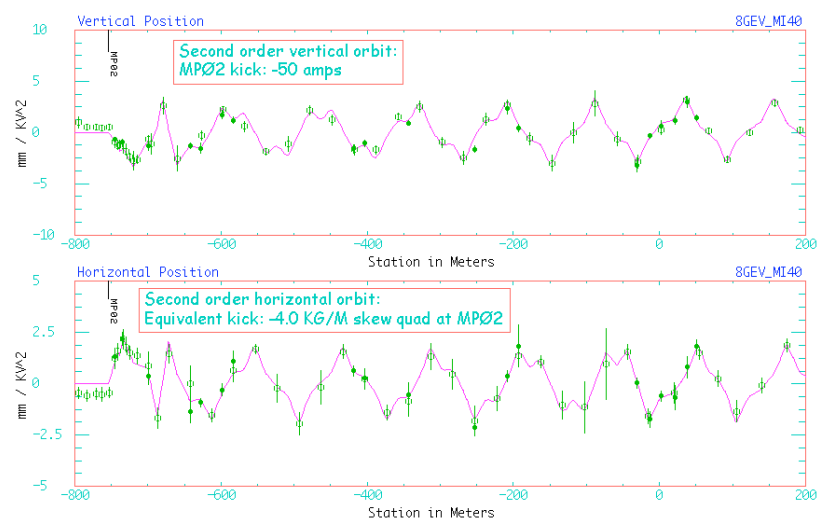

Figure 3. Second order harmonic orbits from MI8 transfer line data. At the bottom is for horizontal plane and top for vertical plane. The extraction septum magnet location is marked by the label on either plots.

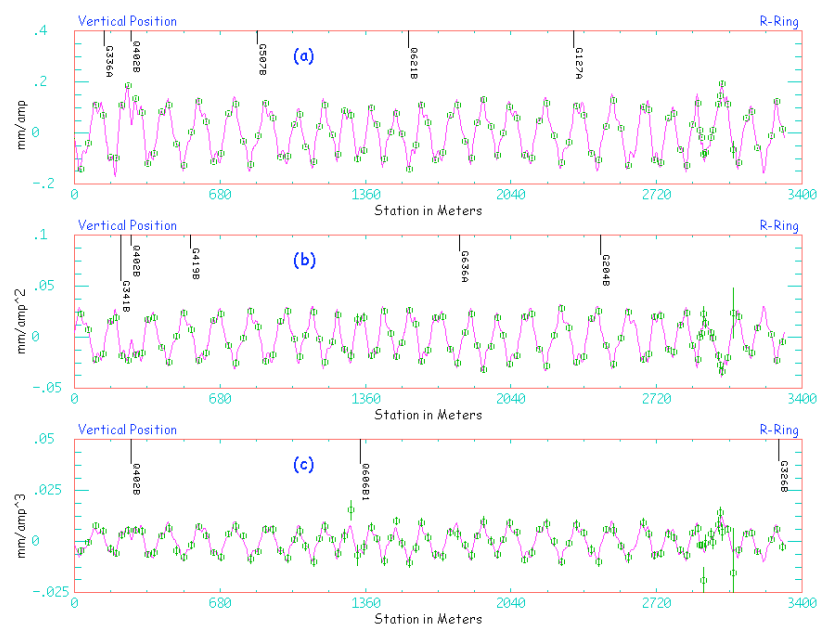

Figure 4. From top are first order (a), second order (b), and third order (c) vertical plane harmonic orbits from Recycler Ring study using horizontal 3-bump at 402 location.

\section{Recycler Ring data}

Figure 4 shows vertical plane harmonic orbits of first, second, and third order from Recycler Ring 3-bump orbit study [2]. These are vertical plane responses to horizontal displacement at 402 location and are the signatures of skew multipole errors. Indicated by labels on each plot are locations found by MICADO/COCU [3] orbit correction procedure to be the likely kick sources. The magenta traces are orbits calculated by modeling program which incorporated these kick sources. Most of these error locations are believed to be fictitious. The use of closed 3bump practically eliminated the effect from harmonic error anywhere outside of the 3-bump region. In all three plots location 402 was implicated as source of kick and this is exactly what the analysis is looking for.

\section{LIMITATIONS}

\section{Resolution}

The effectiveness of harmonic analysis depends on the resolution of position monitors and the magnitudes of error fields. In the MI8 line data shown in Figure 1 the position resolution is in hundred of microns. The corresponding errors in sextupole and skew sextupole field turned out to be quite large and the analysis worked. For Recycler Ring data shown in Figure 2, the position resolution is in the range of 10 microns and the position variation throughout the data set is 100 microns or less. The analysis worked out as well. In fact, the Recycler Ring Lambertson magnets had been suspected of field errors. However, the analysis was not feasible until the upgrade of its BPM system.

\section{Closed orbit stability}

The procedure of decomposition requires a set of orbit data, taken sequentially. This set of position data, one monitor at a time, is fitted to a polynomial of certain order to derive harmonic orbits. Unintended machine orbit variation could bias the fitting algorithm and introduce fictitious harmonic component of any order. This potentially can cause false alarm and confusion. Random orbit motion due to drifting dipole power supply currents is discussed in reference [2].

\section{CONCLUSION}

Harmonic decomposition is a simple concept and has already been shown to be a useful tool in the search for field error. The technique is not by any means the equivalent of a precision field measurement. The ability to see the higher order effect depends on the resolution of BPM system and the stability of the machine orbit. Recent improvement of the Recycler Ring BPM system [4] is a success story both in instrumentation engineering and in its application for orbit data analysis.

\section{REFERENCES}

[1] M.J. Yang, "Multi-pole fields in MP02", Fermilab document, Beams-doc-1573-v1

[2] M.J. Yang, "Harmonic analysis of selected Recycler locations using closed 3-bump orbits.", Fermilab document, Beams-doc-1746-v1

[3] D. Brandt, W. Herr, J. Miles, and R. Schmidt, "A New Closed Orbit Correction Procedure for the CERN SPS and LEP”, Nucl. Inst. and Meth., A293(1990) 305.

[4] Fermilab document RR-BPM-0001.rev1.1 